\title{
Caracterización morfológica de la cápsula ovígera del parásito Caligus rogercresseyi ${ }^{\#}$
}

\author{
Morphological characterization of the egg capsule of the sea louse Caligus rogercresseyi
}

\author{
R Jaramillo ${ }^{\mathrm{a}}$, O Garrido ${ }^{\mathrm{a}}$, G Asencio ${ }^{\mathrm{b}}$, P Barría ${ }^{\mathrm{c}}, \mathrm{J}_{\text {Mancilla }}^{\mathrm{d}}$ \\ anstituto de Ciencias Marinas y Limnológicas, Universidad Austral de Chile, Valdivia, Chile. \\ ${ }^{\text {b} C e n t r o ~ d e ~ I n v e s t i g a c i o n e s ~ I-M a r, ~ U n i v e r s i d a d ~ d e ~ L o s ~ L a g o s, ~ P u e r t o ~ M o n t t, ~ C h i l e . ~}$ \\ ${ }^{c}$ Unidad Caligus, Laboratorio Central, Marine Harvest Chile, Puerto Montt, Chile. \\ dPrograma de Doctorado en Ciencias de la Acuicultura, Universidad Austral de Chile, Valdivia, Chile.
}

\begin{abstract}
SUMMARY
The sea louse, Caligus rogercresseyi, is a marine ectoparasite living on the skin of several salmonid species. Studies on its life cycle have focused mainly on host living stages but no investigations have been conducted regarding the egg, the egg capsule, or its embryonic development. Since the knowledge of early developmental stages of Caligus is scarce, to propose any control measure preventing the parasite reaching its infective stage is difficult. As a way to contribute to the knowledge of the reproductive biology of this parasite, we propose to perform a characterisation of the morphological ultrastructure of the egg capsule using both light and electron microscopy. The light microscopy reveals a capsule wall, built of six main layers, three of which have dark solid bands of color comprised of external, middle and inner dense layers separated by two clear inner and outer layers. All of the five layers are surrounded by an external layer of mucous. Transmission electron microscopy reveals a more complex constitution: the mucus layer corresponds to a triple layer. The dense outer layer consists of four sub layers while the outer clear layer is made up of two distinct sub-layers separated by a thin dark layer; internally there is a middle dense layer. The inner clear layer is $51 \mathrm{~nm}$ at its thinnest portion, but can reach 2.4 microns as part of the wall of the capsule. The innermost layer corresponds to the chorion surrounding the egg.
\end{abstract}

Key words: morphology, egg capsule, sea lice, Caligus rogercresseyi.

\section{RESUMEN}

Caligus rogercresseyi es un ectoparásito marino que habita sobre la piel de varias especies de salmónidos. Los estudios acerca de su ciclo de vida han centrado su atención en las etapas de vida libre, sin que se hayan realizado investigaciones respecto del huevo, de la cápsula ovígera o de su desarrollo embrionario. El desconocimiento de las fases tempranas del desarrollo de Caligus impide proponer medidas de control antes que el parásito logre alcanzar su fase infectante. Como una forma de contribuir al conocimiento de la biología reproductiva de este parásito, nos proponemos realizar una caracterización de la ultraestructura de la cápsula ovígera de Caligus rogercresseyi utilizando la microscopía óptica y electrónica. La MO revela que la pared de la cápsula está formada por seis capas principales: tres corresponden a bandas compactas de coloracion oscura denominadas capa densa: externa, media e interna, las que se encuentran separadas por dos capas claras, llamadas capa clara externa e interna, sobre estas cinco capas se dispone una capa mucosa externa. La MET revela una constitución más compleja: la capa mucosa corresponde a una triple capa. La capa densa externa está constituida por cuatro subcapas mientras que la capa clara externa corresponde a dos subcapas claras separadas por una delgada capa oscura; más internamente se encuentra la capa densa media. La capa clara interna mide $51 \mathrm{~nm}$ en su porción más delgada, pero puede alcanzar $2,4 \mu \mathrm{m}$ cuando forma parte del tabique de la cápsula. La envoltura más interna corresponde al corion que envuelve al huevo.

Palabras clave: morfología, cápsula ovígera, Caligus rogercresseyi.

\section{INTRODUCCIÓN}

Desde sus inicios, la industria salmonera chilena se ha desarrollado en una zona geográficamente reducida generando con ello una notable concentración de la actividad piscícola en la zona sur-austral. Es así como se ha observado un considerable crecimiento de la fase de agua dulce en la Región de La Araucanía, mientras que los procesos de crecimiento y engorda se realizan en aguas interiores del Pacífico Sur principalmente en la Región de Los Lagos con un $40 \%$ de la producción nacional; en la Región de

\footnotetext{
Aceptado: 06.11.2014.

\# Financiado con aportes de Marine Harvest Chile

* Casilla 567, Valdivia, Chile; jjaramil@uach.cl
}

Aysén con un 56,5\% y la Región de Magallanes con 3,5\% (SERNAPESCA 2014)

Como consecuencia de esta segregación, los peces cultivados se han hecho proclives a contraer una amplia variedad de enfermedades, entre las que se cuentan la enfermedad bacteriana del riñón (BKD), la necrosis pancreática infecciosa (IPN), la anemia infecciosa del salmón (ISA), siendo en este momento el síndrome rickettsial salmonídeo (SRS), la enfermedad infecciosa más importante (Rozas y Enríquez 2013).

Junto con la propagación de las citadas enfermedades, las altas densidades de cultivo contribuyen a desarrollar una elevada carga parasitaria en los peces, promoviendo las infecciones de epidemias principalmente como la del piojo del salmón (más conocida como caligidosis), la que 
produce heridas en la piel y depresión inmunológica del pez (Rozas y Asencio 2007, Bravo y col 2008).

El piojo del salmón es conocido en Chile con el nombre de Caligus (Caligus rogercresseyi) (Boxshall y Bravo 2000). Se halla presente en las Regiones de Los Lagos, de Aysén y Magallanes, y afecta principalmente a los salmones cultivados en estas regiones (Asencio y col 2011a). Según Boxshall y Bravo (2000), esta especie es un ectoparásito exclusivamente marino que habita sobre la piel de varias especies de peces cultivados como el salmón del Atlántico (Salmo salar), el Salmón Coho (Oncorhynchus kisutch) y la trucha arco iris (Oncorhynchus mykiss). Asimismo afecta especies de peces silvestres como el róbalo (Eleginops maclovinus), el pejerrey (Odonthestes regia) y el lenguado de ojo chico (Paralichthys microps) (Carvajal y col 1998).

La literatura sugiere que $C$. rogercresseyi fue transmitido desde los peces nativos, principalmente a la trucha arco iris y al salmón del Atlántico que son las especies más susceptibles de ser infectadas no así al salmón Coho que muestra una alta resistencia en iguales condiciones geográficas y ambientales.

Durante su ciclo de vida, $C$. rogercresseyi pasa por tres estados planctónicos (vida libre en la columna de agua) y cinco estados parasitarios (Ritchie y col 1993, Pike y Wadsworth 2000, González y Carvajal 2003), cuya duración depende de la temperatura en la que ocurra el desarrollo, pero siempre dentro del rango de fluctuación natural de temperaturas para la zona en donde se realiza el cultivo de salmones en Chile (González y Carvajal 2003).

La información existente hasta el momento indica que C.rogercresseyi permanece en los hospederos nativos y salmonídeos de cultivo de las Regiones de Los Lagos y de Aysén, aun cuando se realizan grandes esfuerzos para su control. Desde los primeros registros de su presencia hasta hoy se han utilizado prioritariamente estrategias de control farmacológicas relacionadas con la eliminación de los estadios parásitos de la especie (adultos y juveniles) en los centros de cultivo de salmones y truchas (Asencio y Riquelme 2013). Por otra parte, las etapas dispersantes del parásito que muestran hábito planctónico (nauplios y copepoditos) carecen de vías de mitigación por lo que se transforman en focos de nuevas infestaciones en el centro originario y su vecindario (Asencio y col 2011 $1^{\mathrm{b}}$ ). Junto a esto, González y col (2012) describen mayor fecundidad de este parásito en hospederos de cultivo que en peces silvestres, lo que incrementa el problema debido a los altos niveles de producción de salmonídeos en los últimos años.

En relación con los aspectos reproductivos de C. rogercresseyi, Osorio (2006) indica que estos son similares al parásito Lepeophtheirus salmonis (Kroyer 1838) y que el proceso de cópula ocurre solamente una vez en su ciclo de vida, ya que el espermatóforo produce una sustancia cementante que impide a la hembra ser inseminada nuevamente. Posterior a la cópula ocurre la fecundación de los huevos en la espermateca de la hembra, con lo cual se da inicio al desarrollo de los sacos ovígeros al interior de los que ocurre el desarrollo intracapsular del parásito.

Estos antecedentes llevan a plantear la importancia de encontrar metodologías conducentes a reducir la fecundidad de esta especie, ya sea mediante la disminución del número de larvas viables; limitando el desarrollo embrionario o inhibiendo la fecundación interna (Osorio 2006). Sin embargo, los estudios hasta ahora realizados en caligidos han centrado su atención en los estados de vida libre con descripciones muy precisas de estados naupliares y copepoditos (González y Carvajal 2003), sin haber considerado realizar investigaciones relacionadas con el desarrollo embrionario, la formación y características de las envolturas del huevo o de la cápsula ovígera.

Intentar un control de este parásito antes de que alcance la fase infectante de su desarrollo requiere conocer las características del huevo maduro; de las cápsulas ovígeras portadoras de los embriones; así como de las diferentes fases del desarrollo embrionario de esta especie. Es por ello que como una primera contribución al logro de este objetivo, nos proponemos describir la morfología y la ultraestructura de la cápsula ovígera de $C$. rogercresseyi obtenidas a partir de hembras de salmonídeos parasitados.

\section{MATERIAL Y MÉTODOS}

\section{OBTENCIÓN DE PECES INFECTADOS Y DE PARÁSITOS}

Las muestras de Caligus fueron colectadas en tres campañas diferentes realizadas durante agosto y septiembre de 2013 a partir de peces capturados desde las balsas jaulas de centros de engorda localizados en Puchilco, Lincay y Lilicura, Chiloé, Chile.

Los parásitos caligidos fueron extraídos a partir de cada uno de los salmones capturados. En el caso de las hembras ovígeras adultas, estas fueron colectadas desde la piel del pez con la ayuda de pinzas finas para luego colocar los parásitos de cada pez de manera separada en frascos plásticos previamente rotulados. Un grupo de muestras fue destinado a realizar estudios a la microscopía óptica (MO) y por tanto fueron fijadas en formaldehído al $4 \%$, mientras que las restantes se fijaron en una solución de glutaraldehído al 2,5\% en buffer fosfato para estudios de microscopía electrónica de transmisión y barrido. Las muestras así fijadas fueron mantenidas a $4{ }^{\circ} \mathrm{C}$ y trasladadas en frío hasta la Universidad Austral de Chile.

\section{CONFECCIÓN DE CORTES HISTOLÓGICOS}

Las secciones histológicas para microscopía óptica así como el procesamiento de las muestras para microscopía electrónica de transmisión (MET) y barrido (MEB) fueron realizadas en las instalaciones del laboratorio de embriología del Instituto de Ciencias Marinas y Limnológicas de la Universidad Austral de Chile. 
Una vez en el laboratorio, las muestras para $\mathrm{MO}$ fueron mantenidas en formaldehído al 4\%; previo al procesamiento, las cápsulas ovígeras fueron separadas de la hembra mediante el uso de pinzas guardándose solamente las cápsulas. Luego de dos semanas de fijación, las muestras de cápsulas ovígeras fueron procesadas de acuerdo con los protocolos de la técnica histológica tradicional (Humason 1962), obteniéndose tanto secciones transversales como longitudinales de aproximadamente $7 \mu \mathrm{m}$ de espesor, las que fueron teñidas con hematoxilina y eosina.

\section{MICROSCOPÍA ELECTRÓNICA DE TRANSMISIÓN}

En el caso de MET, las muestras fueron lavadas en buffer fosfato de sodio, $\mathrm{pH}$ 7,2-7,4 (0,2 M), posteriormente postfijadas en $\mathrm{OsO}_{4}$ al $1 \%$ en el mismo buffer por un periodo de $90 \mathrm{~m}$ y enseguida procesados para su inclusión en una mezcla de epon-araldita. Secciones finas $(60-90 \mathrm{~nm})$ fueron montadas en grillas de cobre, teñidas con acetato de uranilo y citrato de plomo para luego ser observadas en un MET Philips EM 300.

\section{MICROSCOPÍA ELECTRÓNICA DE BARRIDO}

Las muestras de cápsulas para MEB se lavaron en buffer fosfato de sodio, $\mathrm{pH}$ 7,2-7,4 (0,2 M) y postfijadas en tetróxido de osmio $\left(\mathrm{OsO}_{4}\right)$ al $1 \%$ en el mismo buffer durante $2 \mathrm{~h}$ a $4{ }^{\circ} \mathrm{C}$. Posteriormente, las muestras fueron deshidratadas en soluciones de concentraciones crecientes de alcohol y acetona seguido de secado al punto crítico del $\mathrm{CO}_{2}$ (Hitachi HCP-2 critical point dryer). Las muestras fueron montadas en un portaespécimen para MEB, recubiertas con una película de oro de aproximadamente 10 a $15 \mathrm{~nm}$ de espesor (Ion coater IB-2) y observadas en un microscopio electrónico de barrido LEO-420.

\section{ANÁLISIS DE IMÁGENES}

Las fotografías obtenidas a la MO, MET y MEB fueron utilizadas para realizar un análisis de imágenes que permitió evaluar cuantitativamente las características de los diferentes componentes de la cápsula ovígera, para ello se utilizó el software de análisis de imágenes SigmaScan pro5 (SPSS Inc.).

\section{RESULTADOS}

El estudio reveló que la cápsula ovígera de $C$. rogercresseyi está formada por una cubierta externa delgada que encierra un número variable de huevos en desarrollo, que se encuentran apilados en su interior (figuras 1 y 2). La longitud promedio de las cápsulas ovígeras (figura 3) fue de $2,15 \mathrm{~mm}(\mathrm{n}=20)$; los huevos midieron $260 \mu \mathrm{m}$ de diámetro promedio $(n=80)$ y $68 \mu \mathrm{m}$ de espesor $(n=80)$; en tanto que el número promedio de huevos por cápsula fue de $29( \pm 3,8 \mathrm{n}=20)$.

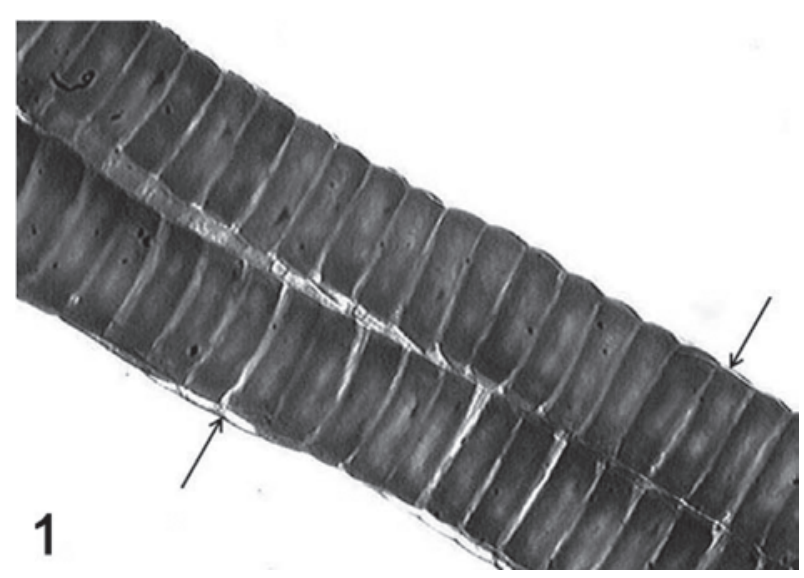

Figura 1. Cápsula de Caligus rogercresseyi, a Microscopía Óptica. (40x), (flechas = embriones en desarrollo.

Caligus rogercresseyi capsule, Light Microscopy (40x), (arrows $=$ developing embryos).

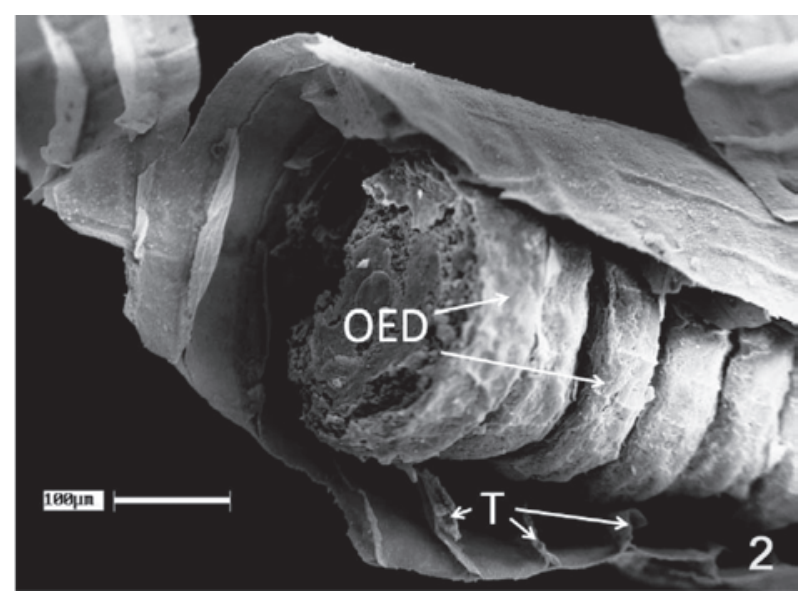

Figura 2. Interior de una cápsula ovígera de Caligus rogercresseyi, a MEB. $\mathrm{OED}=$ ovas en desarrollo, $\mathrm{T}=$ tabiques $)$. barra $=$ $100 \mu \mathrm{m}$

SEM of the inner side of a Caligus rogercresseyi capsule. OED developing embryos, $\mathrm{T}=$ septum.

El estudio a la microscopía óptica reveló que la pared de la cápsula está formada por al menos seis capas diferentes: tres de ellas corresponden a bandas que aparecen definidas como líneas delgadas, pero de carácter compacto y con alta afinidad tintorial que le otorga una coloración oscura a la microscopía óptica (figura 4). Estas capas han sido denominadas capa densa externa, capa densa intermedia y capa densa interna, respectivamente, y se encuentran separadas entre ellas por otras dos capas de baja densidad tintotial, y que aparecen de una tonalidad algo más clara (capa clara externa y capa clara interna).

En el exterior aparece una sexta capa tenuemente definida y que podría corresponder a una capa de tipo mucoso debido a la escasa afinidad tintorial que presenta. Dentro de la misma pared capsular es posible reconocer una 


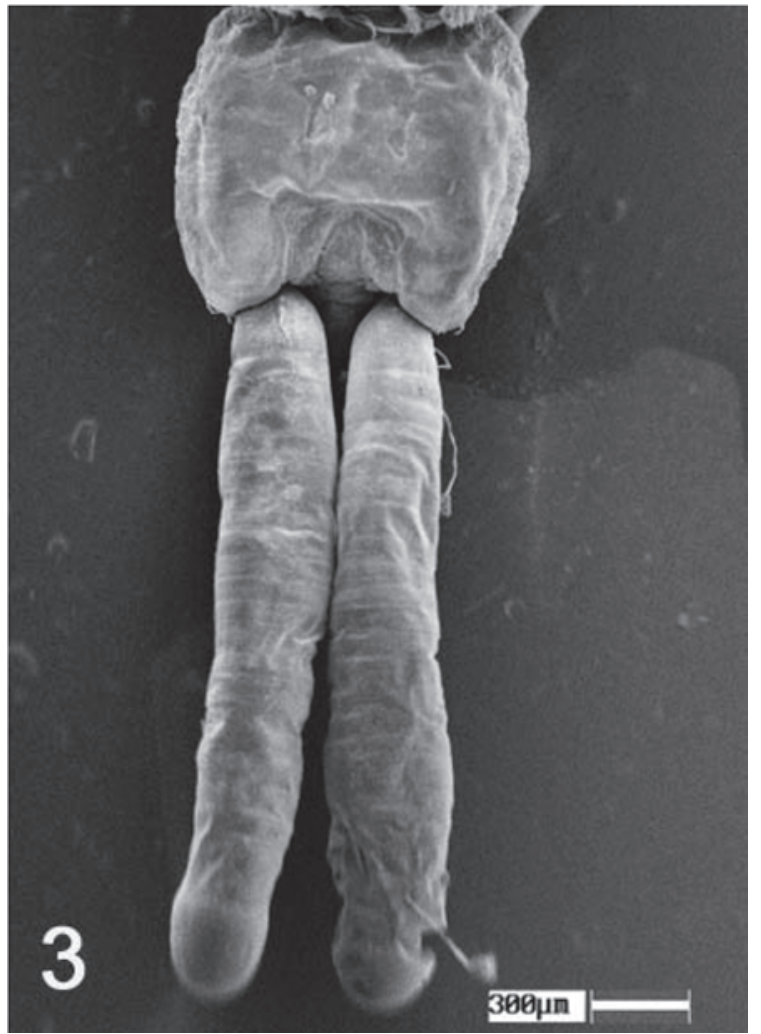

Figura 3. Hembra de Caligus rogercresseyi con cápsulas ovígeras, a MEB. Barra $300 \mu \mathrm{m}$

SEM of a female Caligus rogercresseyi with egg sacs. bar $=300 \mu \mathrm{m}$.

serie de estriaciones definidas por dos líneas de tonalidad oscura (similar a las descritas previamente) que delimitan un pequeño espacio de tonalidad más clara (con apariencia similar a las capas descritas como claras (figura 4)) y dispuestas de manera perpendicular a la dirección en que corren las capas de la pared. Adicionalmente, la cara interna de la pared capsular exhibe una proyección lateral que se dirige hacia la región media de la cápsula generando una especie de borde o tabique que permite separar cada uno de los huevos en desarrollo (figuras 2 y 4).

El análisis de las imágenes obtenidas al MEB permite corroborar la existencia de al menos cinco de las seis capas descritas previamente como parte de la pared de la cápsula ovígera; cada una de estas capas aparece bajo la forma de una doble pared de baja densidad electrónica separadas por una capa intermedia (figura 5) con apariencia de mayor densidad electrónica (más oscura). La cubierta mucosa externa no aparece revelada, probablemente porque pudo ser arrastrada durante el proceso de preparación de la muestra.

La pared tiene un espesor promedio de $1,15 \mu \mathrm{m}$ mientras que cada una de las capas de doble pared miden en promedio $0,42 \mu \mathrm{m}$ espesor, la capa intermedia mide 0,3 $\mu \mathrm{m}$ en promedio (figura 5). La doble capa interna de la pared capsular emite una proyección hacia el interior de la cápsula cada $70 \mu \mathrm{m}$ generando un pequeño reborde o

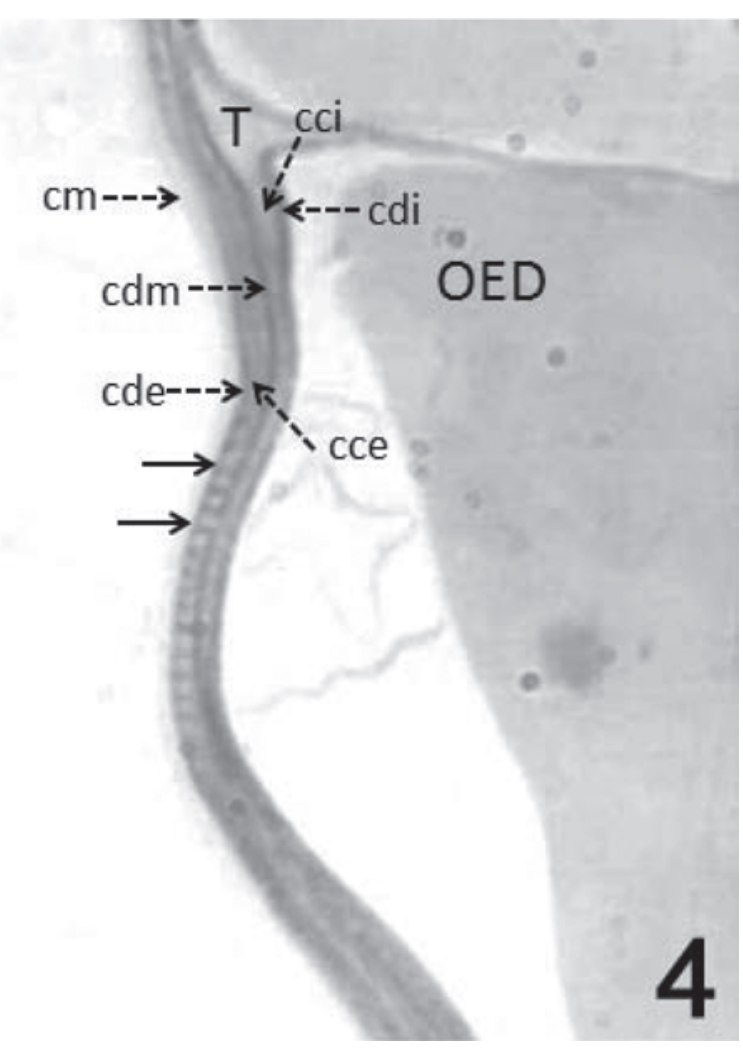

Figura 4. Sección longitudinal de la pared de la cápsula de Caligus rogercresseyi, a la Microscopía Óptica (1000x) barra 300 $\mu \mathrm{m} ; \mathrm{T}=$ tabique, $\mathrm{OED}=$ ova en desarrollo; $\mathrm{cm}=$ capa mucosa; cce = capa clara externa; cci $=$ capa clara interna; cde = capa densa externa; cdi $=$ capa densa interna; $\mathrm{cdm}=$ capa densa media; flechas $=$ estriaciones.

Lateral view of capsule wall of Caligus rogercresseyi, Light Microscopy $(1000 \mathrm{x})$. OED = developing embryos, $\mathrm{T}=$ septum; $\mathrm{cm}=$ mucous layer; $c c e=$ clear outer layer capa; $c c i=$ clear inner layer; cde $=$ dark outer layer; $c d i=$ dark inner layer; $c d m=$ dark middle layer; arrows $=$ furrows.

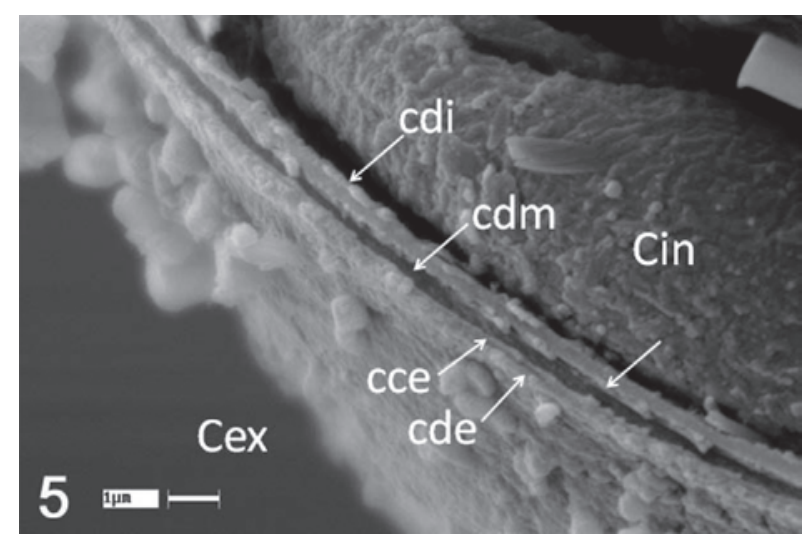

Figura 5. Pared de la cápsula ovígera de Caligus rogercresseyi, a MEB. Cex = cara externa; Cin = cara interna; cee = capa clara externa; cci $=$ capa clara interna; cde $=$ capa densa externa; cdi = capa densa interna; cdm = capa densa media.

SEM of capsule wall of Caligus rogercresseyi. $\mathrm{Cex}=$ external side Cin $=$ inner side ; ce $=$ clear outer layer capa cci $=$ clear inner; cde $=$ dark outer layer; $\mathrm{cdi}=$ dark inner layer; $\mathrm{cdm}=$ dark middle layer. 
tabique de aproximadamente $18 \mu \mathrm{m}$ de longitud promedio y que permite la separación entre embriones en desarrollo (figura 6).

El estudio de la pared de la cápsula a la MET revela una constitución algo más compleja que la descrita previamente, puesto que si bien se reconocen las seis capas ya citadas; desde el punto de vista ultraestructural cada una de estas aparece organizada por una serie de láminas más delgadas o subcapas que forman parte de cada una las seis capas principales.

Externamente se confirma la existencia de una capa mucosa de $50 \mu \mathrm{m}$ en promedio (figura 4 ), pero que corresponde a una triple capa: una capa mucosa externa seguida de una capa mucosa media de dimensiones similares (140 $\mathrm{nm}$ y $120 \mathrm{~nm}$ respectivamente) y una capa mucosa interna de $300 \mathrm{~nm}$. Estas tres capas se originan como resultado del depósito de algunas fibras mucosas dispuestas en sentido paralelo a la cápsula y otras en sentido transversal al eje de la cápsula. Esta disposición antiparalela de las fibras contribuye a entregar la apariencia de tricapa (figura 7). Del mismo modo, la capa densa externa $(210 \mathrm{~nm})$ está constituida por cuatro subcapas: dos de ellas delgadas: la primera de baja densidad electrónica seguida de una alta densidad electrónica, para luego continuar con otras dos capas levemente más gruesas que las anteriores: una de baja densidad, seguida de una cuarta más densa electrónicamente (figura 7). En este caso las capas tienen una apariencia fibrosa y la característica de mayor o menor densidad electrónica está relacionada con la disposición antiparalela de las fibras descrita previamente para las otras capas.

La capa clara externa $(310 \mathrm{~nm})$ está constituida por dos subcapas de baja densidad electrónica, separadas por una de alta densidad; más internamente se encuentra la capa densa media de $0,34 \mu \mathrm{m}$ de espesor y cuya constitución está dada por la presencia de fibras dispuestas en un solo sentido.

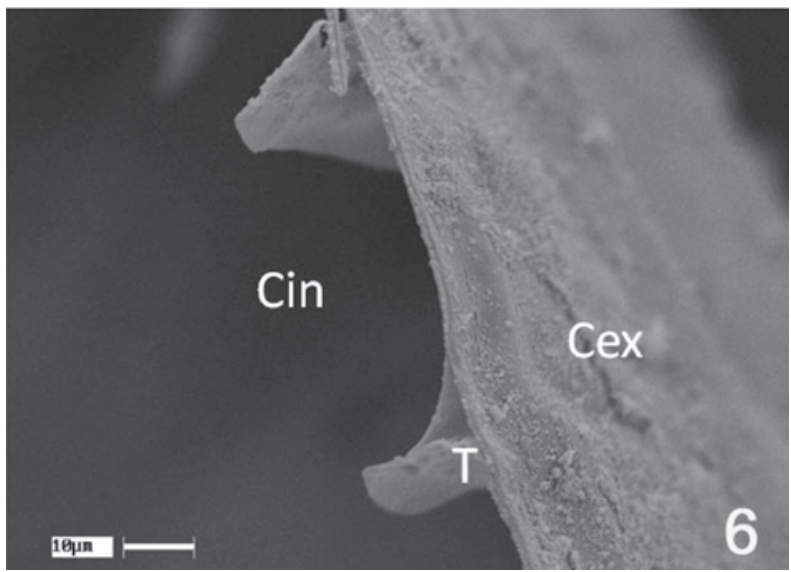

Figura 6. Pared de la cápsula ovígera de Caligus rogercresseyi con trabéculas, a MEB. Cex = cara externa; Cin = cara interna; $\mathrm{T}=$ trabécula.

SEM of capsule wall of $C$ rogercresseyi showing a septum. Cex $=$ outer side; Cin $=$ inner side; $\mathrm{T}=$ septum.
La capa clara interna es una monocapa de $51 \mathrm{~nm}$ de espesor en su porción más delgada, pero puede alcanzar fácilmente los 2,4 $\mu \mathrm{m}$ de ancho cuando se le observa formando parte del tabique que separa los embriones (figuras 4, 6 y 8). Esta capa está formada por fibras muy compactas y orientadas paralelamente entre ellas, a diferencia de la estructura observada en la zona de los tabiques, en donde las fibras se disponen de manera entrelazada y algo más laxas que en la zona más delgada de la cápsula.

La envoltura más interna de la cápsula corresponde a una capa muy electrodensa de $91 \mathrm{~nm}$ de espesor y situada próxima a la membrana plasmática del huevo, esta capa se ha denominado capa densa interna (figura 7).

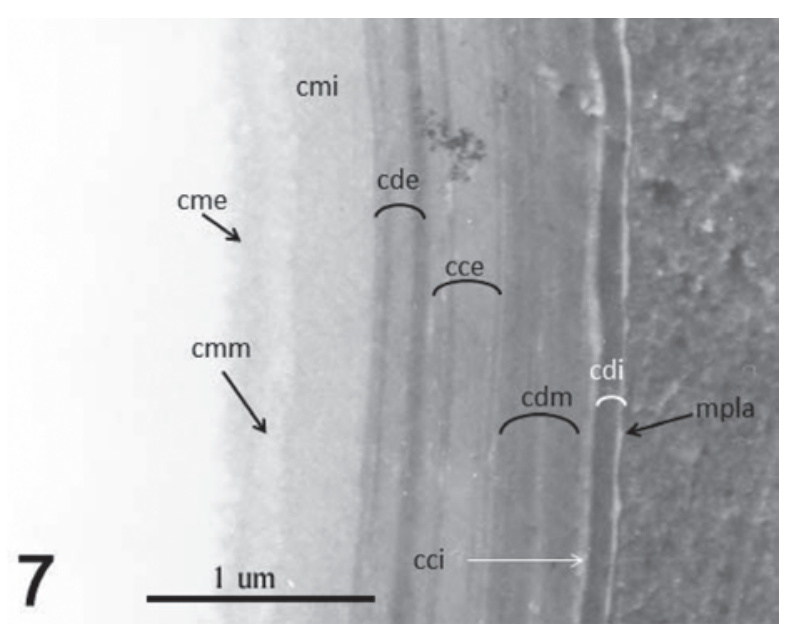

Figura 7. Sección transversal de la pared de la cápsula ovígera de Caligus rogercresseyi, a MET. cme = capa mucosa externa; $\mathrm{cmm}=$ capa mucosa media $; \mathrm{cmi}=$ capa mucosa interna; $\mathrm{cce}=$ capa clara externa; cci $=$ capa clara interna; cde = capa densa externa; cdi = capa densa interna $;$ cdm = capa densa media; $\mathrm{mpla}$ = membrana plasmática del ovocito.

TEM of a cross section of capsule wall of Caligus rogercresseyi . $\mathrm{cme}=$ outer mucous layer $; \mathrm{cmm}=$ middle mucous layer; $\mathrm{cmi}$ = inner mucous layer; cce = clear outer layer capa; cci = clear inner; cde $=$ dark outer layer; cdi $=$ dark inner layer; $\mathrm{cdm}=$ dark middle layer.

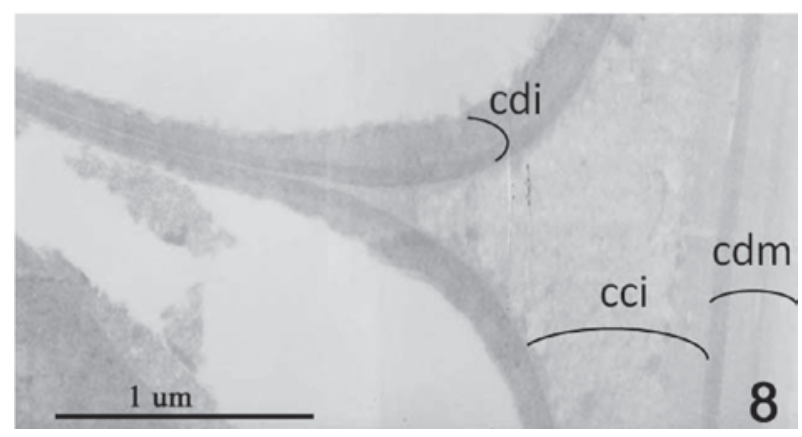

Figura 8. Pared de la cápsula ovígera de Caligus rogercresseyi con trabéculas a MET. cci = capa clara interna; cdi = capa densa interna; $c d m$ = capa densa media.

TEM of capsule wall of Caligus rogercresseyi showing a septum . cci $=$ clear inner; cdi $=$ dark inner layer; cdm $=$ dark middle layer. 


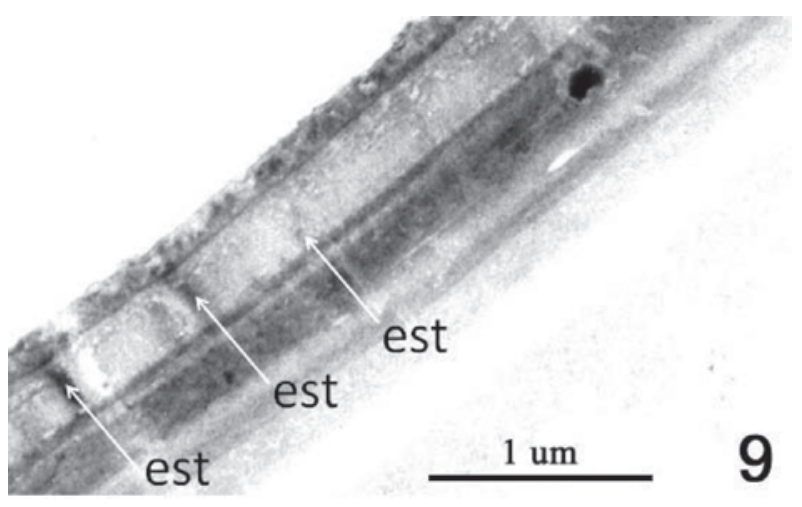

Figura 9. Pared de la cápsula ovígera de Caligus rogercresseyi con estriaciones a MET. est = estriaciones.

TEM of capsule wall of Caligus rogercresseyi showing furrows. est $=$ furrows .

Las estriaciones descritas a la MO para la pared de la cápsula fueron difíciles de encontrar en las secciones para MET, no obstante, evidencias de su presencia aparecen reveladas bajo la forma de pequeñas áreas altamente condensadas y muy electrodensas (figura 9).

\section{DISCUSIÓN}

Desde el punto de vista morfológico, la cubierta externa de la cápsula ovígera de $C$. rogercresseyi es similar a la descrita para Lepeoptheirus mugiloides, Lepeoptheirus salmonis, Pseudocaligus fugu y otros caligidos (Ohman y Townsend 1998, Venmathi Maran y col 2011, Levicoy 2014). La longitud aquí reportada para la cápsula fue de 2,2 mm y está dentro de los rangos descritos por González y col (2012) quienes indican que esta fluctúa entre $1,5 \mathrm{~mm}$ y 6,1 mm de longitud total. Del mismo modo, el número promedio de huevos reportados en este estudio $(n=29)$ se encuentra dentro del rango de fecundidad descrita para la especie y que oscila entre 14 huevos y 59 huevos para cápsulas que fluctúan entre 1,5 mm y 6,1 mm de longitud (González y col 2012).

Muchas especies de invertebrados marinos encapsulan sus huevos como una manera de proteger a los juveniles de los efectos de la predación y de la potencial mortalidad causada por la dispersión de las larvas en el plancton; dichas cápsulas pueden ser estructuras gelatinosas o rígidas dependiendo del material con el que se fabriquen (Thorson 1946). Estudios acerca de la composición química de la pared de cápsulas rígidas (descritas para neogastrópodos entre otras especies) indican que estas están constituidas por glicoproteínas, glicógeno o lípidos (Miloslavich 1996), dispuestos de manera antiparalela y que se organizan bajo la forma de un número variable de capas (Bayne 1968). Los resultados aquí descritos nos muestran que la cápsula ovígera de $C$. rogercresseyi está formada por una serie de capas dispuestas de manera muy similar a la descrita para neogastrópodos (Thorson 1935, Matthews-Cascon y col
2010) y por tanto puede ser clasificada como una cápsula de tipo rígido.

Hasta el momento no existen estudios que describan la composición química de la cápsula ovígera de los caligidos, la única mención al respecto es aportada por Carvajal y col (2001) quienes al estudiar la relación simbiótica entre $U$ donella sp y $C$. rogercresseyi indican que la cutícula de esta se muestra ligeramente acidófila al ser teñida con hematoxilina, mientras que la subcutícula presenta basofilia reactiva solo a $\mathrm{pH} 4,5$. Del mismo modo, dichos autores señalan que la cápsula ovígera del copépodo sirve como zona de fijación del parásito y que en dicha zona aparece una banda de tinción metacromática que corresponde a glicosaminoglicanos ácidos.

Los resultados aquí descritos para la cubierta de la cápsula ovígera de $C$. rogercresseyi y que muestra la presencia de capas de alta densidad electrónica seguidas por capas de baja densidad electrónica, podrían ser explicadas por la alternancia en la disposición de las fibras proteicas que forman las diferentes capas de la cápsula ovígera. Esta disposición alternada de las fibras podría explicar la presencia de bandas de coloración diferente descritas a partir de las secciones histológicas teñidas con hematoxilina y eosina usadas en este trabajo y a su vez podría explicar la afinidad tintorial diferente descrita por Carvajal y col (2001) para este mismo tipo de cápsulas.

El huevo de $C$. rogercresseyi al igual que el de Lepeophtherius salmonis presenta dos capas (Pietrak 2002); una primera envoltura de apariencia densa con alta densidad electrónica que corresponde a una matriz extracelular o envoltura externa del huevo, estructura común a todos los huevos y que se denomina corion, seguida de una delgada membrana plasmática que delimita el citoplasma del huevo. El corion así descrito para Lepeophtherius salmonis por Pietrak (2002) corresponde a la capa densa interna aquí reportada y que por efectos de corte aparece adherida a la superficie interna de la cápsula ovígera de $C$. rogercresseyi.

El estudio de ciclo de vida de cualesquier especie requiere del conocimiento de todas las etapas del ciclo partiendo por el proceso de fecundación y terminando en el momento en que los individuos alcanzan la madurez sexual e incluyendo las características del sistema reproductor de la especie. Respecto de $C$. rogercresseyi, los estudios de su ciclo de vida se han centrado fundamentalmente en los estadios de vida libre y en los estadios parásitos, sean estos fijos o móviles puesto que son dichas fases los principales agentes causantes de la infección de los peces, pero olvidando las fases más tempranas del desarrollo intracapsular (embrionarios) y que son las que conducen al desarrollo de los estadios infectantes.

Los resultados aquí descritos respecto de la ultraestructura de la cápsula ovígera de $C$. rogercresseyi constituyen los primeros antecedentes respecto de la organización y características de la pared de esta cápsula que facilita el crecimiento y desarrollo de los estadios embrionarios de este 
parásito, junto con ello, los antecedentes aquí reportados permiten visualizar un campo de investigación hasta ahora no explorado y que eventualmente pudiese contribuir al eventual control del ciclo de vida de este parásito.

\section{AGRADECIMIENTOS}

Los autores desean agradecer la colaboración de Marine Harvest Chile por facilitar el material biológico utilizado en el estudio; así como también su contribución económica para llevar a cabo esta investigación.

\section{REFERENCIAS}

Asencio G, J Carvajal, MT González, C Molinet, V Riquelme. 2011ª. Análisis de los descansos productivos coordinados en el control de la caligidosis en Chile. FishfarmingXpert 1, 44-51.

Asencio G, S Marín, R Ibarra, P Miranda. 2011 ${ }^{\text {b }}$. La Caligidosis en salmónidos de cultivo; resultados del programa de monitoreo, vigilancia y control de Caligus rogercresseyi de Intesal. Período octubre 2007-diciembre 2009. Compendio Intesal.

Asencio G, V Riquelme. 2013. Análisis histórico de las estratégias de vigilancia y control de la caligidosis en la salmonicultura chilena. SalmonXpert 1, 43-49.

Bayne CJ. 1968. Histochemical studies on the egg capsules of eight gastropod molluscs. Proc Malacol Soc Lond 38, 199-212.

Boxshall GA, S Bravo. 2000. On the identity of the common Caligus (Copepoda: Siphonostomatoida: Caligidae) from salmonid netpen system in southern Chile. Contrib Zool 69, 137-146.

Bravo S, V Pozo, MT Silva. 2008. The tolerance of Caligus rogercresseyi to salinity reduced in Southern Chile. B Eur Assoc Fish Pat 28, 198-206.

Bron JE, AP Shinn, C Somerville. 2000. Ultrastructure of the cuticle of the chalimus larvae of the salmon louse Lepeoptheris salmonis (Kroyer, 1837) (Copepoda: Caligidae). Contrib Zool 69, 39-49.

Carvajal J, L González, M George-Nascimento. 1998. Native sea lice (Copepoda: Caligidae) infestation of salmonids reared in netpen systems in southern Chile. Aquaculture 166, 241-246.

Carvajal J, G Ruiz, F Sepúlveda. 2001. Symbiotic relationship between Udonella sp. (monogenea) and Caligus rogercresseyi (Copepoda), a parasite of the Chilean rock cod Eleginops maclovinus. Arch Med Vet 33, 31-36.

González L, J Carvajal. 2003. Life cycle of Caligus rogercresseyi (Copepoda: Caligidae) parasite of Chilean salmonids. Aquaculture 220, 101-117.

González MT, B Arenas, G Asencio, C Molinet, J Carvajal. 2012. Fecundity of sea louse Caligus rogercresseyi on native host Eleginops maclovinus, and farmed salmon in Southern Chile. Aquac Res 43, 853-860.

Guzmán AJ. 2010. Eficacia del tratamiento con Benzoato de Emamectina contra el parásito. Caligus rogercresseyi en el salmón del atlántico (Salmo salar) y su relación con factores de tipo ambiental y de manejo. Memoria de título, Escuela de Medicina Veterinaria, Universidad Austral de Chile, Valdivia, Chile.
Helgesen KO, S Bravo, S Sevatdal, J Mendoza, TE Horsberg. 2014. Deltamethrin resistance in the sea louse Caligus rogercresseyi (Boxhall and Bravo) in Chile: bioassay results and usage data for antiparasitic agents with references to Norwegian conditions. Jour Fish Dis doi:10.1111/jfd.12223

Humason H. 1962. Animal Tissue Techniques. W.H. Freeman and Company, San Francisco, USA.

Levicoy CG. 2014. Estudio histológico de las estructuras reproductivas de dos géneros de ectoparásitos caligidos de Eleginops maclovinus en el sur de Chile. Memoria de título, Escuela de Biología Marina, Universidad Austral de Chile, Valdivia, Chile.

Matthews-Cascon H, C Rocha-Barreira, PE Penchaszadeh, G Bigatti. 2010. Description of egg capsules of Voluta ebraea Linnaeus, 1758 (Gastropoda:Neogastropda). Com de la Soc Malac del Uruguay 9, 237-244.

Miloslavich P. 1996. Biochemical composition of prosobranch egg capsules. J Mollus Stud 62, 133-139.

Ohman MD, AW Townsend. 1998. Egg strings in Euchirella pseudopulchra (Aetideidae) and comments on constraints on egg brooding in planktonic marine copepods. J Mar Syst 15, 61-69.

Osorio V. 2006. Conducta de apareamiento y reproducción del ectoparásito Caligus rogercresseyi (Boxshall \& Bravo 2000) (Copepoda: Caligidae) en el hospedador Eleginops maclovinus (Valenciennes, 1840) (Pisces: Nototheniidae). Memoria de título, Escuela de Biología Marina, Universidad de Los Lagos, Puerto Montt, Chile.

Pietrak M. 2002. Evaluation of three potential methods for preventing the spread of the salmon louse Lepeophtheirus salmonis (Kroyer, 1837) Thesis Master of Science (in Marine Biology), The Graduate School of The University of Maine, Maine, USA.

Pike AW, SL Wadsworth. 2000. Sealice on salmonids: Their biology and control. Academic Press Inc., San Diego, USA, Pp 233-337.

Ritchie G, AJ Mordue, AW Pike, H Rae.1993. The reproductive output of Lepeophtheirus salmonis adult females in relation to seasonal variability of temperature and photoperiod. In: Boxshall GA, Defaye D (eds). Pathogens of wild and farmed fish: sea lice. Ellis Horwood. Chichester, UK, Pp 153-165.

Rozas M, G Asencio. 2007. Assessment of Epidemiologic Situation of Caligiasis in Chile: Toward to Effective Control Strategy. Salmo Ciencia 43-59.

Rozas M, R Enríquez. 2013. Piscirickettsiosis and Piscirickettsia salmonis in fish: a review. Jour Fish Biol 37, 163-188.

Sernapesca, Servicio Nacional de Pesca, Chile. 2014. Informe técnico nueva estrategia de programa de control de caligidosis, unidad de salud animal. Santiago, Chile.

Thorson G. 1935. Studies on the egg-capsules and development of Artic marine prosobranchs. Medd om Gronland 100, 1-71.

Thorson G. 1946. Reproduction and Larval Development of Danish Marine Bottom Invertebrates. Medd kömm Havundersög Kbh plankton 4, 523.

Venmathi Maran BA, S Ohtsuka, I Takami, S Okabe, G Boxshall. 2011. Recent advances in the biology of the parasitic copepod Pseudocaligus fugu (siphonostomatoida Caligidae) host specific to pufferfishes of the genus Takifugu (Actynopterigii, Tetraodontidae). In: Asakura A, Fransen C (eds). New Frontiers in Crustacean Biology; Koninklijke Brill NV, Leiden, Netherlands, Pp 31-45. 